\title{
Gravity Constraints on the Measurements of the Speed of Light
}

\section{Faiçal Ramdani}

Laboratory of Geophysics \& Natural Hazards, Geophysics, Natural Patrimony \& Green Chemistry Research Center (Geopac), Mohamed V University, Rabat, Morocco

Email: faicalramdani@gmail.com

How to cite this paper: Ramdani, F. (2019) Gravity Constraints on the Measurements of the Speed of Light. International Journal of Astronomy and Astrophysics, 9, 265-273.

https://doi.org/10.4236/ijaa.2019.93019

Received: June 23, 2019

Accepted: September 2, 2019

Published: September 5, 2019

Copyright (c) 2019 by author(s) and Scientific Research Publishing Inc. This work is licensed under the Creative Commons Attribution International License (CC BY 4.0).

http://creativecommons.org/licenses/by/4.0/

(c) (i) Open Access

\begin{abstract}
The speed of light in a vacuum is a constant of special relativity, electromagnetic wave theories, and astrophysical distances. However, several measurements of its speed $(c)$ at locations on the Earth's surface seem to vary at different times during the last century. Efforts have been made on instruments performance to achieve a unique viable value in any spacetime referential. The time-variability on $c$-values obtained is here addressed inside the gravity field $(g)$ in which the measurements of $c$ have been estimated. It appears a correlation of $c$ and $g$ both daily (tidal) and yearly (no-tidal) variations which suggest that the gravity acceleration control the $c$-variability everywhere in a spacetime referential. Implications of this model provide a sensitivity constant of $c$ from $g$, and the estimates of $c$ on planets of the solar system where $g$ values are known. It is deduced an upper limit of gravity in black hole that can cancel the speed of light in the horizon.
\end{abstract}

\section{Keywords}

Gravity, Time, Speed of Light, Solar System, Black Hole

\section{Introduction}

Velocity of light was intensively studied in the last century since it was a basis for Special relativity and electromagnetic waves theories. A variety of instruments have been used to improve the accuracy of $c$-measurements [1]. Efforts were focused on reducing errors due to instruments and to immediate environments such as temperature, pressure, clocks, length of ray path which was fully discussed as possible sources for no regular values obtained from the speed of light. However, no identical $c$-values are maintained [2] despite the fact that the same instruments are used under the same laboratory conditions. To solve these issues 
most studies computed the average of a set of measurements, and proposed it as a final result of the speed of light [1] [3] [4]. This was done at several epochs from the beginning of the last century until the last value of $c$ (1983) accredited by the International bureau of Weight and length which in the way defined the meter from the adopted $c$-value $(299,792,458 \mathrm{~m} / \mathrm{s})$. Such relativistic parameter has direct implications on the accuracy of space geodetic techniques [5]. Models of variable speed of light (VSL) and anisotropy were developed later to explain cosmological issues [6] [7]. A review of the $c$-fluctuations [2] [8] where data are available shows that the values of $c$ change but not in an arbitrary form, and may be re-found after a certain time. This variation of $c$ is small and has not been of special interest. A systematic decreasing $\mathrm{c}$ with time has not been successfully achieved [9] since varying instruments were used on varying epochs, and because of further observations have indicated an increasing value in the same laboratory. The impact of gravity on velocity of light has not been fully studied, but its possible existence was, however, raised since 1911 [10]. The problematic connection of gravity and speed of light is addressed as it appears complex when time variation is included (Figure 1). The variable $\mathrm{g}$ is of a low level ( $\mu$ gal) due to tidal forces and ocean loading, and about 1 mgal due to tectonic processes, while spatial variations in gravity are observed between inland and offshore zones, plate boundaries, Mountains and plateau areas, even using similar instruments to distant areas [11].

\section{Experimental Data}

Absolute gravity data from several regions provided by worldwide data centers, BGI (Bureau Gravimetrique International) and NOAA (National Oceanic and Atmospheric Administration) show the spatial variations of gravity. Figure 2(a) shows three years of annual variations of short and long wavelengths related to tidal and no-tidal forces in Boulder station. The variation is periodic and the average trend is there horizontal but it can be decreasing in other sites as in a

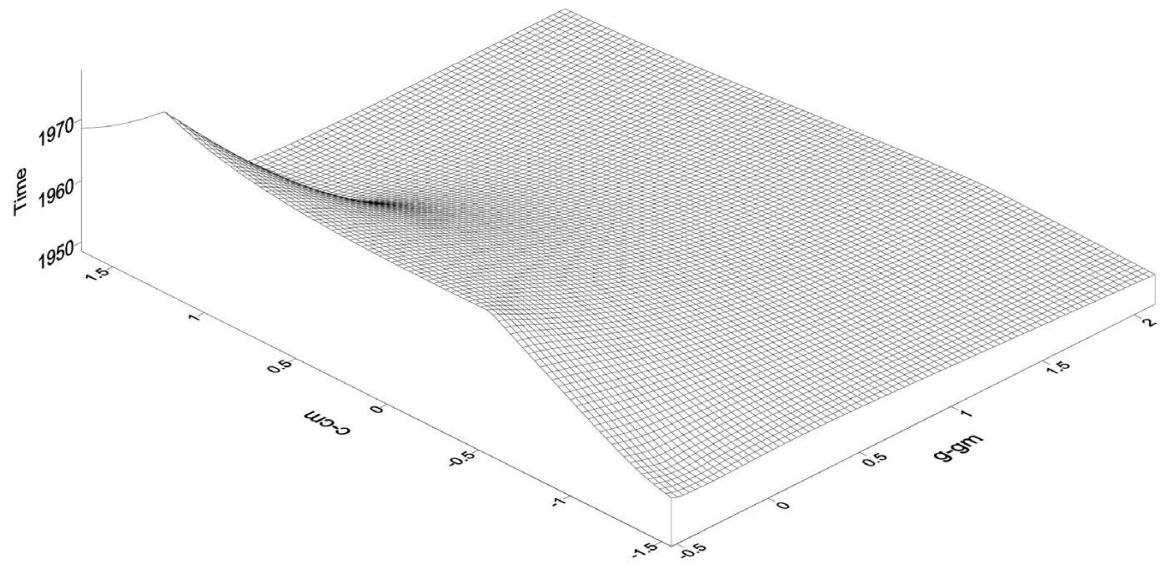

Figure 1. 3D-deviations of $c$ and $g$ from $\mathrm{cm}$ and $g m$ values. Absolute $g$-values are observed in the site of $c$-measurements but not synchronized with $c$-values. The time is assigned to $c$-measurements. 


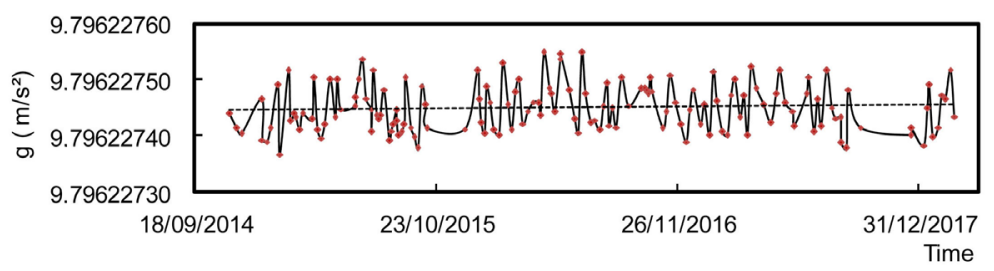

(a)

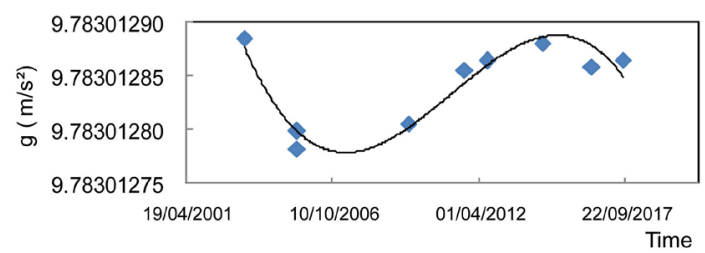

(b)

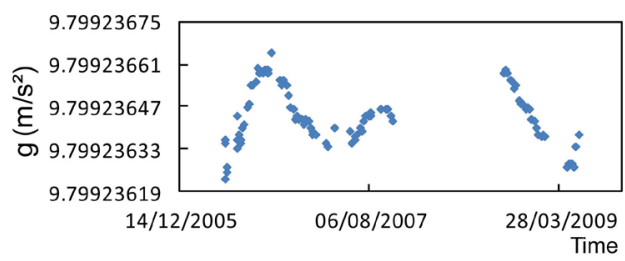

(c)

Figure 2. Yearly variations of the absolute gravity is shown in varying setting, Boulder (a) where the trend curve (dashed line) is horizontal, Darwin Australia observations with a line of trending variation (b) and in Conception (Chile) which shows evidence for periodic variation at large period of gravity observations (c).

Norway experiment [12]. Much longer period of observations with limited data (Figure 2(b)) shows again oscillatory variation in Darwin station (Australia) and even with a large number of observations as in Conception-Chile where the underlying subduction made it low the gravity anomaly (Figure $2(\mathrm{c})$ ). Speeds of light measurements were made without taking into account the state of gravity in the area of $c$-experiments. When several measurements are achieved, and it can last several days or weeks, the gravity has also changed due to tidal forces, atmospheric and ocean loading. Resulting successive $c$-values are therefore of prime importance, not the average of the values obtained from the experiment. While each $c$-value may be affected by flow in clocks, the variation between values is not clock-dependent. As no synchronized values of $c$ and $g$ are available since 1911 when equipment of such experiment did not exist, there are, however, some successive experiments of $c$-estimates. Since successive they are a function of time in the same site. It is, therefore, possible to compare the evolution of $c$ with time and the absolute gravity recorded in the same site. Variation model appears in the $c$-fluctuations by using two sets of measurements, one due to Froomer [13] and other measurements carried out in Boulder (US bureau of standard) during two weeks [14]. The variation seems to be periodic to both cases (Figure 3(a) and Figure 3(b)) which vary around a $c$-mean. The $c$-values found in Chile where low gravity is known provided high average of $3.05 \times 10^{8}$ $\mathrm{m} / \mathrm{s}$ [15]. The accuracy of this value is not of importance here, but the distances 


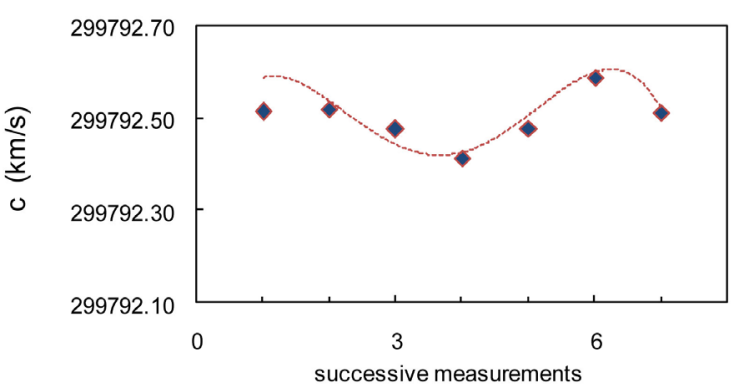

(a)

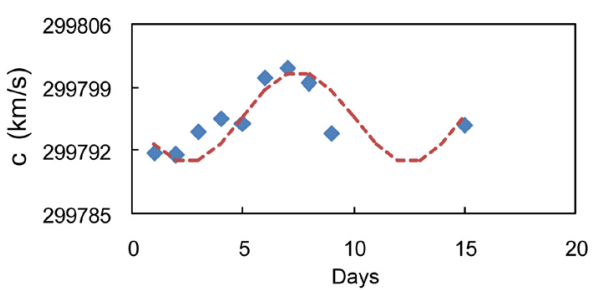

(b)

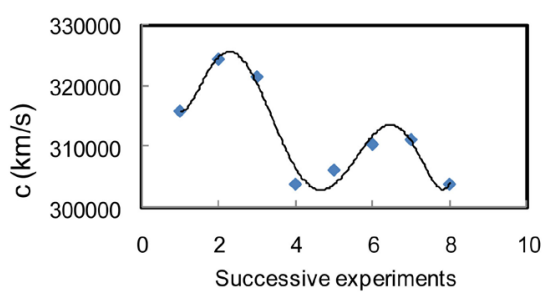

(c)

Figure 3. Successive c-measurements in NPL (UK) (a) and US Boulder station (b) and Chile (c). Trend variations by $c$-fluctuations appear to be periodic.

and time delay of their successive observations, then in other terms the time-variations of $c$. Figure 3(c) shows the results of distance-times measurements and it also fits with a periodic trend. Other high value of $c$ estimated in Caracas experiments $\left(3.009 \times 10^{8} \mathrm{~m} / \mathrm{s}\right)$ using one-way method. [16] agrees with low gravity environment but it lacks the accuracy of the two-way method. More reliable set of $c$-measurements in Sweden. [17] shows daily variations of $c$ that is of tidal type when compared morning and afternoon observations (Figure 4(a) and Figure 4(b)). During the 11 days of measurements appears a peak to peak period about 3 - 4 days the origin of which is open to question in the absence of synchronized gravity data in the same site of c-measurements.

\section{Gravity-Time-Velocity Model}

As $c$-values vary with time g also vary, and the variation seems to be periodic. This suggests that the speed of light is not affected by gravity by bending only, but the value of speed also is sensitive to the time and place where it is measured. These examples show the form of variability in the values of both $c$ and $g$ around $c_{0}$ and $g_{0}$. The amount of sensitivity of $c$ from gravity may be experimented. The correlation of their variability (not the values) suggests a relationship between $c$ and $g$ in term of causality. The fluctuations in $\mathrm{c}$ are assumed to be due to gravity 


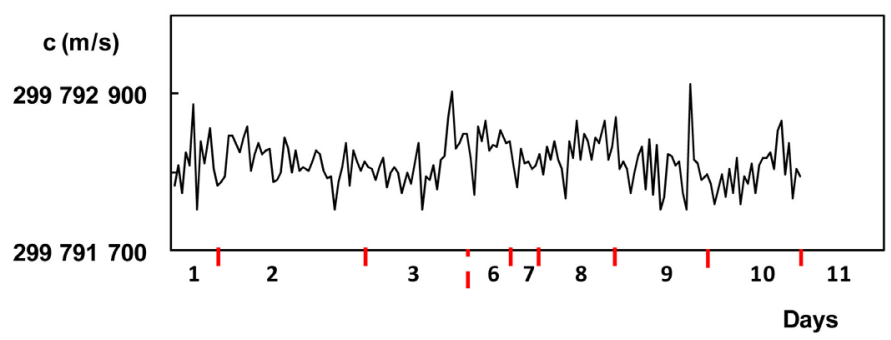

(a)

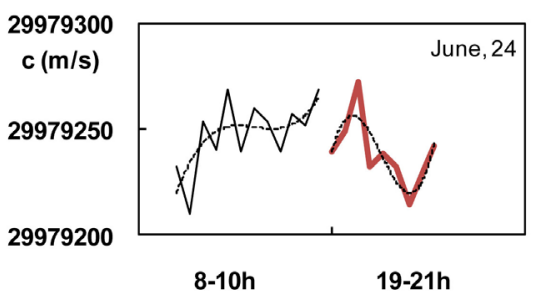

(b)

Figure 4. Daily variations of c-estimates from Sweden with a peak to peak period of about 3 - 4 days are indicated in (a); variation during one day between morning and afternoon is shown in (b) where a trend variation is indicated (dashed lines).

which is time varying. In this simple model gravity is the acceleration that affects time variation of $c$.

$$
c(g, t)=A \cdot g(t)+c_{0}
$$

where $A$ is constant (sec). Experimental data of $c$ using geodimeter and Laser methods [3] [4] [8] are compared with absolute gravity data measured in the same site (Figure 5(a) and Figure 5(b)). Since $c$ estimates are taken as average of several measurements, they are then representative for the site where an absolute gravity is observed (Table 1). Time variations of $g$ and then of $c$ in the same site is here assumed to be of second order. Since the values determined by geodimeter methods are unreliable because of the large margin of errors (Figure $5(\mathrm{a})$ ), it is more appropriate to take as values those determined by Laser whose errors appear acceptable (Figure 5(b)). The trend of the $c_{L}$ values can be expressed by the equation

$$
C_{L}=-50.446 g+299792953.5
$$

where $C_{0}=299,792,953.5 \mathrm{~m} / \mathrm{s}$ is the $c$-velocity in vacuum without gravity,

$$
C_{0}-C=50.44 g=\Delta C
$$

where $\Delta C$ is the variation of $c$ in a gravitational field $g$.

In this relationship, it appears the sensitivity $A$ of $c$ when $g$ varies. $A$ is too small to be significant in Earth surface measurements where the range of variation of $g$ is limited $\left(9.76-9.83 \mathrm{~m} / \mathrm{s}^{2}\right)$. When gravity varies substantially in outer space, such a relationship can gain more attention. Table 2 shows updated values of $c$ in varying gravity field of solar planets while a black hole upper limit gravity value $g_{B H}$ is obtained for $c=0$ since at more higher values of $g_{B H}$ this will make $c$ negative according to Equation (2). 


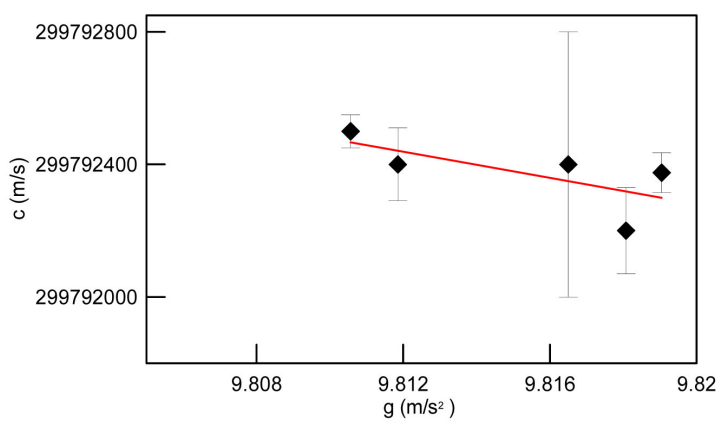

(a)

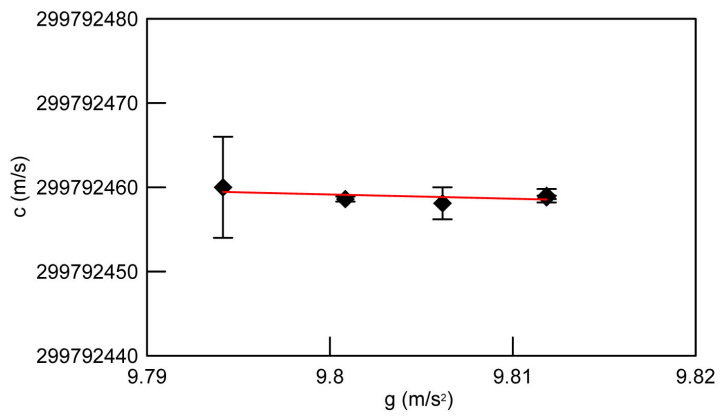

(b)

Figure 5. $C$-variations versus gravity velocity $(\mathrm{g})$ is plotted in varying sites at times of $c$-measurements using geodimeter (a) and laser (b) methods. Resulting trending curves are decreasing to both cases.

Table 1. $C$-measurements at varying epochs using geodimeter and laser and associated estimates of absolute gravity recorded in the site of $c$-observations.

\begin{tabular}{ccccc}
\hline Methods & Time & $C(\mathrm{~m} / \mathrm{s})$ & $C$-error $(\mathrm{m} / \mathrm{s})$ & $g\left(\mathrm{~m} / \mathrm{s}^{2}\right)$ \\
\hline Geodimeter & 1953 & $299,792,400$ & 110 & 9.811856 \\
& 1953 & $299,792,200$ & 130 & 9.818074 \\
& 1955 & $299,792,400$ & 400 & 9.816501 \\
& 1967 & $299,792,500$ & 50 & 9.810565 \\
Laser & 1971 & $299,792,375$ & 60 & 9.819047 \\
& 1972 & $299,792,460$ & 6 & 9.794161 \\
& 1974 & $299,792,459$ & 0.8 & 9.811856 \\
& 1978 & $299,792,458.8$ & 0.2 & 9.811856 \\
& 1979 & $299,792,458.1$ & 1.9 & 9.80616 \\
& 1983 & $299,792,458.6$ & 0.3 & 9.800849 \\
\hline
\end{tabular}

$$
g_{B H}=5943218.401 \mathrm{~m} \cdot \mathrm{s}^{-2}
$$

With near 0-velocity the photons cannot escape from black holes, or probably are accumulated in the horizon. The upper limit of $c_{\max }$ from which $c$ slowly decreases by a constant $A$ due to change in gravity may be reached at $g \approx 0$.

$$
c_{\max }=299792953.5 \mathrm{~m} \cdot \mathrm{s}^{-1} ; A=-50.44
$$


The difference between $c$-adopted (1983) and $c_{\max }$ is $495.51 \mathrm{~m} / \mathrm{s} . c_{\max }$ is the speed in a vacuum without gravity. The variation of $c$ is inversely proportional to gravity, which induces that velocity increases at low gravity field at a fixed time. In turn, when gravity is big then the speed of light is reduced, and may be vanished at infinite gravity environment as it is the case around black holes. Figure 6 shows the decreasing values of $c$ in the solar system, the slower speed is found in the sun because of its high gravity.

Table 2. Results of $c$-estimates in planets of solar system where $g$-values (Nasa) are known, and gravity limit in black hole.

\begin{tabular}{ccc}
\hline Planet & $g\left(\mathrm{~m} / \mathrm{s}^{2}\right)$ & $c(\mathrm{~m} / \mathrm{s})$ \\
\hline Sun & 274 & $299,779,132.9$ \\
Jupiter & 23.1 & $299,791,788.3$ \\
Neptune & 11 & $299,792,398.7$ \\
Saturn & 9 & $299,792,499.5$ \\
Earth & 9.8 & $299,792,459.2$ \\
Uranus & 8.7 & $299,792,514.7$ \\
Venus & 8.9 & $299,792,504.6$ \\
Mars & 3.7 & $299,792,766.9$ \\
Mercury & 3.7 & $299,792,766.9$ \\
Moon & 1.6 & $299,792,872.8$ \\
Pluto & 0.7 & $299,792,918.2$ \\
Black hole & $5,943,555.78$ & 0 \\
\hline
\end{tabular}

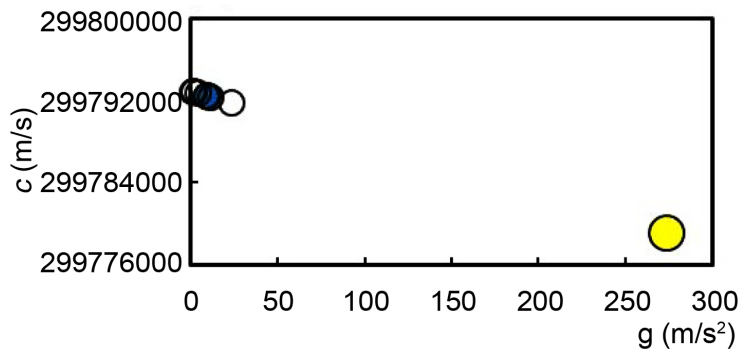

(a)

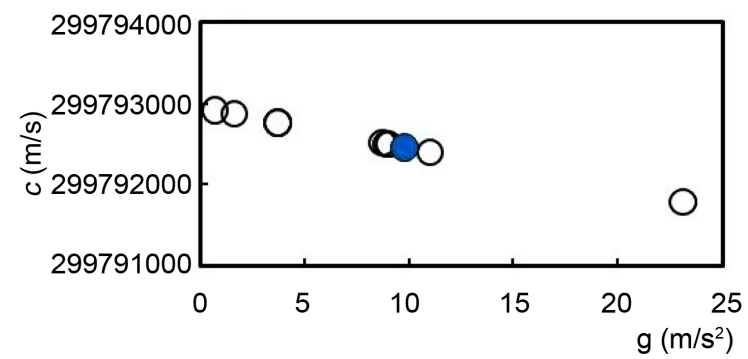

(b)

Figure 6. Speed of light fluctuations model in the solar system with sun (a); and without sun (b) based on gravity shows linear decreasing of the speed of light from solar planets including earth (full blue circle) with respect to sun (yellow). 


\section{Discussion and Conclusions}

The spacetime variation of $c$ explains the issues in the last century to obtain a unique value even when similar instruments were used. The varying spatial sites where $c$ was estimated in addition to the timing of the observation constrained the comparison between results. Even though this study used limited but the more reliable available values of $c$ and gravity, it has introduced a control of gravity in electromagnetic wave propagation in term of space-time fluctuation of c. A synchronized observation of gravity and $c$-velocity may confirm the hypothesis while the use of the average of consecutive $c$-measurements has no physical significance. Each determination is a natural value of $c$ at a given time and at a given value of gravity which is also space dependent. Since gravity changes on Earth are periodic in the short term, long term tectonic factors can cause a variation of gravity in the site of $c$-measurements. Observations from Figure 4 exhibit the short-term daily $c$-variation that may be caused by short term gravity variation. For this reason, the use of the means of $c$-values may be valid in a particular site because of the periodicity of $g$, but the c-values may change in another site when gravity is different. Measuring the speed $c$ in the Moon may confirm if the suggested $c$-value (Table 2) is valid and if the coefficient $A$ is universal or planet-dependent. Second-order variation due to time should be introduced to avoid excessive use of averages. This will result in a spatio-temporal variation of $c$ and a global mapping of $c$ which proves to be necessary as it exists for $g$. The correlation between $c$ and $g$ could be highlighted in a referential of time. This will lead to better decipher the degree of sensitivity of $c$ when $g$ varies both daily and yearly. Laser methods suggest the subtraction of about $50 \mathrm{~g}$ from $c_{\max }$ to determine a realistic value of $c$. In black hole setting, no need to cancel time to stop photons from moving in the horizon, the limit value $g_{B H}$ seems sufficient. Speed fluctuation of $c$ may improve the model of c-invariant while the exact impact of $g$ on $c$ needs further synchronized measurements of $(c, g, t)$ parameters. By introducing the impact of gravity on the speed of light the constancy of many parameters will be refined.

\section{Conflicts of Interest}

The author declares no conflicts of interest regarding the publication of this paper.

\section{References}

[1] Aslakson, C.I. (1964) The Velocity of Light. International Hydrographic Review, Monaco, 41, 69-83.

[2] Prokhonik, J. and Morris, W.T. (1993) A Review of Speed of Light Measurements since 1676. Journal of Creation (Former CEN Technical Journal), 7, 181-183.

[3] Baird, K.M., Smith, D.S. and Whitford, B.G. (1979) Confirmation of the Currently Accepted Value 299792458 Meter per Second for the Speed of Light. Optics Communications, 31, 367-368. https://doi.org/10.1016/0030-4018(79)90216-5 
[4] Evenson, K.M., Wells, J.S., Petersen, F.R., Danielson, B.L., Day, G.W., Barger, R.L. and Hall, J.L. (1972) Speed of Light from Direct Frequency and Wavelength Measurements of the Methane-Stabilized Laser. Physical Review Letters, 29, 1346-1349. https://doi.org/10.1103/PhysRevLett.29.1346

[5] Muller, J., Soffel, M. and Klioner, S.A. (2008) Geodesy and Relativity. Journal of Geodesy, 82, 133-145. https://doi.org/10.1007/s00190-007-0168-7

[6] Magueijo, J. (2003) Cosmology "without" Constants. Astrophysics and Space Science, 283, 493-503. https://doi.org/10.1023/A:1022560802810

[7] Cahill, R.T. (2006) A New Light-Speed Anisotropy Experiment: Absolute Motion and Gravitational Waves Detected. Progress in Physics, 4, 73-92.

[8] Evenson, K.M. (1975) The Development of Direct Optical Frequency Measurement and the Speed of Light. ISA Transactions, 14, 209-216. https://doi.org/10.7567/JJAPS.14S1.65

[9] Barrow, J.D. (2005) Cosmological Constants and Variations. Journal of Physics. Conference Series, 24, 253-267. https://doi.org/10.1088/1742-6596/24/1/031

[10] Einstein, A. (1911) On the Influence of Gravity on the Propagation of Light. Annalen der Physik, 35, 898-908. https://doi.org/10.1002/andp.19113401005

[11] Hinderer, J., Crossley, D. and Xu, H. (1994) A Two Year Comparison between the French and Canadian Superconducting Gravimeter Data. Geophysical Journal International, 116, 252-266. https://doi.org/10.1111/j.1365-246X.1994.tb01796.x

[12] Memin, A., Rogister, Y., Hinderer, J., Omang, O.C. and Luck, B. (2011) Secular Gravity Variations at Svalbard (Norway from Ground Observations and Grace Satellite Data). Geophysical Journal International, 184, 1119-1130. https://doi.org/10.1111/j.1365-246X.2010.04922.x

[13] Froome, K.D. (1958) A New Determination of the Free-Space Velocity of Electromagnetic Waves. Proceedings of the Royal Society of London. Series A, Mathematical and Physical Sciences, 247, 109-122. https://doi.org/10.1098/rspa.1958.0172

[14] Florman, E.F. (1955) A Measurement of the Velocity of Propagation of Very-High-Frequency Radio Waves at the Surface of the Earth. Journal of Research of the National Bureau of Standards, 54, 335. https://doi.org/10.6028/jres.054.038

[15] Ortiz, M. and Montecinos, A.M. (2015) How to Measure the Speed of Light with a Dinner Budget. Revista Brasileira de Ensino de Física, 37, 1502. https://doi.org/10.1590/S1806-11173711649

[16] Greaves, E.D., Rodriguez, A.M. and Ruiz-Camacho, J. (2009) A One Way Speed of Light Experiment. American Journal of Physics, 77, 894-896. https://doi.org/10.1119/1.3160665

[17] Bjerhammar, A. (1972) A Determination of the Velocity of Light Using the Twin Superheterodyne Principle. Tellus, 24, 481-495. https://doi.org/10.3402/tellusa.v24i5.10661 